Schultheiß, Birgit; Obstoj, Peter; Maiwald, J.; Henning, Günter:

Monitoring zur Erforschung der Ursachen dialyseassozierter und dialyseinduzierter Arrhythmien

\footnotetext{
Zuerst erschienen in: $\quad$ Biomedizinische Technik = Biomedical Engineering. - Berlin [u.a.] : de Gruyter. - 47 (2002), S1b, S. 732-735.

Jahrestagung der Deutschen Gesellschaft für Biomedizinische Technik (DGBM) im VDE ; 36 (Karlsruhe) : 2002.09.25-27

Erstveröffentlichung: 2002

Datum Digitalisierung: $2009-11-26$

ISSN (online): $\quad$ 1862-278X

ISSN(print) 0013-5585

DOI: $\quad$ 10.1515/bmte.2002.47.s1b.732

[Zuletzt gesehen: 2019-12-12]
}

„Im Rahmen der hochschulweiten Open-Access-Strategie für die Zweitveröffentlichung identifiziert durch die Universitätsbibliothek IImenau."

"Within the academic Open Access Strategy identified for deposition by IImenau University Library."

„Dieser Beitrag ist mit Zustimmung des Rechteinhabers aufgrund einer (DFGgeförderten) Allianz- bzw. Nationallizenz frei zugänglich."

„This publication is with permission of the rights owner freely accessible due to an Alliance licence and a national licence (funded by the DFG, German

Research Foundation) respectively."

\section{DFG}

Nationallizenzen 


\title{
MONITORING ZUR ERFORSCHUNG DER URSACHEN DIALYSE- ASSOZIIERTER UND DIALYSEINDUZIERTER ARRHYTHMIEN
}

\author{
B. Schultheiß ${ }^{1}$, P. Obstoj ${ }^{1}$, J. Maiwald ${ }^{2}$, G. Henning ${ }^{1}$ \\ ${ }^{1}$ Institut für Biomedizinische Technik und Informatik, Technische Universität Ilmenau, Deutschland \\ ${ }^{2}$ Klinik für Innere Medizin IV, Friedrich-Schiller Universität Jena, Deutschland \\ birgit.schultheiss@tu-ilmenau.de
}

\begin{abstract}
Zur Untersuchung der Entstehungsursachen dialyseinduzierter bzw. -aggravierter Arrhythmien erfolgte ein impedanzkardiographisches Monitoring sowie die Bestimmung der Serumelektrolyte. Bei 60,6\% der Dialysebehandlungen, in denen das Auftreten klinisch relevanter Arrhythmien beobachtet wurde, konnte eine deutliche Zunahme von VES bzw. SVES während der Dialysebehandlung festgestellt werden. Neben der durch den Flüssigkeitsentzug bedingten, erheblichen Kreislaufbelastung lagen die Ursachen offenbar in zu stark reduzierten Plas$m a-K^{+}-K o n z e n t r a t i o n e n$. Eine unmittelbar mit Dialysebeginn einsetzende und über die gesamte Behandlung anhaltende Extrasystolie bzw. absolute Arrhythmie wurde insbesondere bei kardial vorgeschädigten Patienten beobachtet. Anhand impedanzkardiographisch ermittelter Kreislaufparameter war in diesen Fällen eine Hauptursache in einem zu hohen bzw. zu schnellen Flüssigkeitsentzug zu sehen.
\end{abstract}

Keywords-Arrhythmie, Extrasystolen, Dialyse, Monitoring

\section{Einleitung}

Die Hämodialysebehandlung ist prädestiniert für das Auftreten oder die Verschlechterung von Herzhythmusstörungen. Etwa 50\% der Todesfalle bei Dialysepatienten sind kardiovaskulär bedingt [1] und ca. 10\% der Fälle des plötzlichen Herztodes sind vermutlich auf Arrhythmien zurückzuführen. Eine Hauptursache dieser Problematik liegt in der erheblichen, dialysebedingten Herz-Kreislaufbelastung bei den oftmals multimorbiden Patienten, die ein hohes Risiko für das Auftreten kardiovaskulärer Komplikationen während der Dialysebehandlung darstellt.

Bisher bleiben supraventrikuläre (SVES) und ventrikuläre (VES) Extrasystolien bzw. Arrhythmien klinisch meist unbemerkt oder sie werden erst bei auftretenden Symptomen erkannt. $\mathrm{Da}$ diese Herzrhythmusstörungen jedoch insbesondere bei kardial vorgeschädigten Patienten zu erheblichen kardiovaskulären Komplikationen führen können, ist einerseits eine kontinuierliche Überwachung von Risikopatienten wünschenswert, andererseits besteht die klinische Notwendigkeit, die patientenindividuell zur Arrhythmieentstehung beitragenden Faktoren detaillierter zu analysieren.

Die Ursachen von während der Hämodialysebehandlung vermehrt auftretenden Arrhythmien werden in der Literatur kontrovers diskutiert, vorrangig scheinen dabci jedoch Störungen des Elektrolythaushaltes (vor allem Hyper- brw. Hypokaliämie) [2], linksventrikulärc Dysfunktion [1], linksventrikuläre Hypertrophic [3], artericlle Hypertonic
[4] sowie die im Alter [1] bzw. mit der Dauer der Dialysebehandlung zunehmende Häufigkeit kardialer Erkrankungen als arrhythmiefördernde bzw. -auslösende Faktoren zu gelten. Vermutlich begünstigt auch der Flüssigkeitsentzug während der Dialyse, welcher eine nicht unerhebliche Kreislaufbelastung für den Patienten darstellt, die Ausbildung von Arrhythmien.

Ziel dieser Arbeit ist es, die Ursachen intradialytisch auftretender Arrhythmien detaillierter zu erforschen. Dazu wurde ein kontinuierliches Kreislaufmonitoring durchgeführt.

\section{Materialien und Methoden}

Zur Untersuchung der Entstehungsursachen intradialytisch auftretender Arrhythmien wurde während 269 Hämodialysebehandlungen bei insgesamt 150 Patienten ein impedanzkardiographisches Monitoring durchgeführt. Die Signalerfassung und -analyse von EKG und IKG erfolgte mit dem Messsystem multiscreen (medis GmbH Ilmenau, Abtastfrequenz 200Hz).

Zur Online-Erkennung von intermittierenden absoluten Arrhythmien wurde das pRR50-Verfahren eingesetzt [5]. Weiterhin erfolgte anhand von Amplituden- und Formparametern aus dem EKG eine online-Vorklassifikation von ventrikulären Extrasystolen, welche nach Beendigung der Dialysebehandlung mittels PatternAnalyse interaktiv validiert wurde. Eine Bewertung der klinischen Relevanz detektierter ventrikulärer Extrasystolien wurde gemäß Lown-Klassifikation vorgenommen, nach der eine Häufigkeit von mehr als 30 VES/ $h$, die Entstehung bestimmter Rhythmen (Bigeminus, Trigeminus) sowie das Auftreten höhergradiger VES (Couplets, Triplets, Salven) als diagnostisch relevant eingestuft werden.

Zur Analyse des zeitlichen Verlaufes der VES wurde die Anzahl der pro 10min-Zeitintervall aufgetretenen VES dargestellt. Durch diese gegenüber der bisher verwendeten Verfahrensweise (stündliche Beurteilung) erhöhte zeitliche Auflösung wird eine exaktere Zuordnung zu klinischen Messwerten bzw. Ereignissen ermöglicht. Um eine ggf. auftretende blutdruckbedingte Arrhythmieentstehung zu erfassen, wurden Blutdruchmessungen ebenfalls in 10-minütigen Abständen durchgeführ.

Zur detaillierteren Erforschung der Fntstchungsursachen von Arrhythmien wurden dic impedanzkandiographisch bestimmten hämodynamischen Parameter (c.H. I jehthonsfraktion und Cardiac Output), der Blutdruck. die wahrend 
der Hämodialyscbchandlung gemessenen Serumelektrolytkonzentration $\left(\mathrm{Na}^{+}, \mathrm{K}^{+}\right.$und $\left.\mathrm{Ca}^{2+}\right)$ sowic vorliegende Befunde aus Routincuntersuchungen von Langreit-EKG und lichokardiographic herangezogen.

Die untersuchte Patientengruppe umfasste 150 Dialysepaticnten (77 männlich, 73 weiblich) und wics mit 63,7 \pm 12,7 Jahren ein relativ hohes Durchschnittsalter auf. Dieses bedingt u.a. den großen Anteil multimorbider Patienten mit relevanten kardiovaskulären Begleiterkrankungen. So belinden sich in der Gruppe beispielsweise 67 Diabetiker, 97 Ilypertoniker (Schweregrad II bzw. III nach WHO) und 46 Patienten mit koronarer Herzkrankheit. Bei 30 Patienten wurde cine permanente absolute Arthythmie festgestellt. Dic Dialysethcrapie erfolgte als 3 bis 5,5-stündige Standard-Bicarbonat-Dialyse. Die angewendete Ultrafiltrationsstrategie wurde patientenindividuell ausgewählt, wobei die mittlere UF-Rate auf $\max .1000 \mathrm{ml} / \mathrm{h}$ begrenzt wurde.

\section{Ergebnisse}

Bei 66 (24.5\%) der analysierten Hämodialysebehandlungen wurden klinisch relevante ventrikuläre Arrhythmien (Lown II - IVb) beobachtet. Diese traten bei $72,1 \%$ der Patienten de novo auf. Weiterhin wurde eine während der Dialyse aufgetretene intermittierende absolute Arrhythmie bei Vorhofflimmern bei 11 Patienten festgestellt.

Die maximale bei einem Patienten innerhalb einer Stunde detektierte Anzahl von VES betrug 1223. Bei dem gleichen Patienten traten während der gesamten Dialysebehandlung (4h) 153 Couplets und 36 Triplets auf.

In der Tabelle 1 werden das mittlere Alter sowie die Verteilung von Hypertonie und linksventrikulärer Hypertrophie der Patientengruppen mit bzw. ohne klinisch relevante ventrikuläre Arrhythmien gegenübergestellt.

Tabelle 1: Vergleich der Patientengruppen hinsichtlich arrhyth mieprädisponierender Faktoren

\begin{tabular}{lccc}
\hline Patientengruppe & $\begin{array}{c}\text { Mittleres } \\
\text { Alter }\end{array}$ & $\begin{array}{c}\text { Hyper- } \\
\text { tonie }\end{array}$ & $\begin{array}{c}\text { Hyper- } \\
\text { trophie }\end{array}$ \\
\hline $\begin{array}{l}\text { Pat. mit ventrikulä- } \\
\text { ren Arrhythmien }\end{array}$ & $69,7 \pm 9,9 \mathrm{~J}$. & $67,4 \%$ & $55,8 \%$ \\
$\begin{array}{l}\text { Pat. ohne ventriku- } \\
\text { läre Arrhythmien }\end{array}$ & $58,9 \pm 14,9$ J. & $61,1 \%$ & $37,6 \%$ \\
\hline
\end{tabular}

Abb. 1 zeigt beispielhaft für einen Patienten (76 Jahre, Diabetes mellitus IIb, art. Hypertonie WHO II, koronare Herzkrankheit) die Herzrate (HR), die Anzahl aufeinanderfolgender VES (1: singuläre VES, 2: Couplet, 3: Triplet) sowie die VES-Häufigkeit pro $10 \mathrm{~min}$-Intervall. Weiterhin sind für die in der Abbildung markierten Zeitpunkte Ausschnitte aus dem aufgezeichneten EKG dargestellt. Es ist deutlich zu erkennen, dass im dargestellten Fall ca. 12 min nach Beginn der Dialysebehandlung schlagartig VES auftreten. Diese Extrasystolen sind auch in der Darstellung der Herzrate zu erkennen. Die Anzahl der detektierten VES nimmt zunächst kontinuierlich zu, erreicht nach ca. 100 min ein Plateau und nimmt nach Dialyseende wieder ab. Insgesamt wurden während der Behandlung 2512 singuläre VES, 97 Couplets und ein Triplet beobachtet.

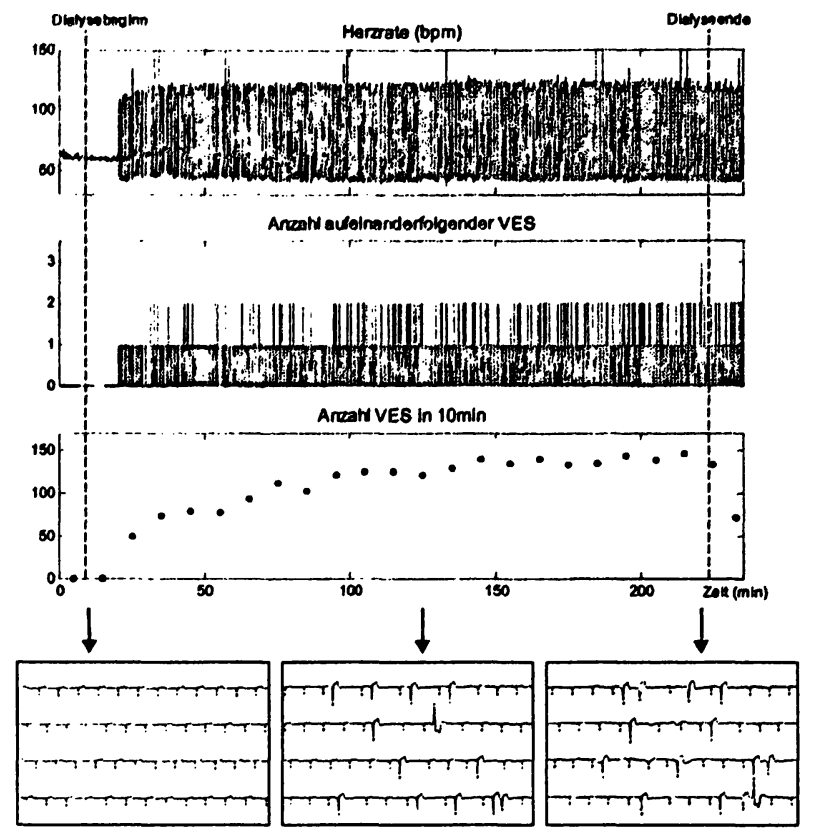

Abbildung 1: Dialyseverlauf mit zunehmender Anzahl von VES und Couplets

Zur Analyse möglicher Arrhythmieursachen wurde zunächst der zeitliche Verlauf des Auftretens von VES betrachtet. Bei den 66 Behandlungen, in denen klinisch relevante ventrikuläre Arrhythmien auftraten, wurde folgender tendenzieller Verlauf der VES-Häufigkeit beobachtet:

- Bei 40 Behandlungen (60,6\%) stieg die Anzahl der VES im Dialyseverlauf deutlich an. Dabei erfolgte die $\mathrm{Zu}$ nahme relativ kontinuierlich über die gesamte Behandlungsdauer oder relativ schnell zu einem gegebenen Zeitpunkt innerhalb des letzten Drittels der Dialysezeit.

- Bei 5 Dialysen (7,6\%) nahm die VES-Häufigkeit von einem relativ hohen Wert zu Beginn kontinuierlich bis zum Behandlungsende ab oder es trat eine deutliche Häufung nur innerhalb des ersten Drittels der Dialysebehandlung auf.

- In 11 Fällen $(16,7 \%)$ trat eine Erhöhung der VESAnzahl im Verlauf der Behandlung auf, wobei die Werte zu Beginn und am Ende deutlich unter diesem Maximum lagen.

- Bei 10 Dialysen (15,2\%) blieb die VES-Anzahl während der Behandlung relativ konstant. In 4 dieser 10 Fälle war dabei jedoch die VES-Häufigkeit während der Dialyse deutlich höher als in den dialysefreien Messabschnitten.

Die bei über der Hälfte der Behandlungen mit klinisch relevanten ventrikulären Arrhythmien beobachtete deutliche Zunahme von VES im Dialyseverlauf legt nahe, dass diese dialyseassoziert bzw. -aggraviert auftreten.

In den Abbildungen 2 und 3 ist beispielhaft der zeitliche Zusammenhang zwischen den Kreislaufparametern Ejektionsfraktion (EF) und Blutdruck sowie der im Dialyseverlauf aufgetretenen Anzahl an VES dargestellt.

Bei der in Abb. 2 gezeigten Dialysebehandlung eines 72jährigen Patienten (arterielle Hypertonie WHO II, linksventrikuläre Hypertrophie, paVK Std. IV) nimmt kurz 
nach Behandlungsbeginn die EF infolge der Ultrafiltration (UF-Rate $1000 \mathrm{ml} / \mathrm{h}$ ) schnell ab und sinkt nach ca. $21 \mathrm{~min}$ unter den Wert von 35\%. Während in der ersten Stunde der Behandlung nur 4 VES auftraten, steigt die Anzahl im weiteren Verlauf deutlich an und erreicht nach einer Messzeit von ca. $200 \mathrm{~min}$ ein Maximum von 58 VES pro $10 \mathrm{~min}$. Nach Dialyseende steigt die EF wieder an und auch die Anzahl der VES ist rückläufig.

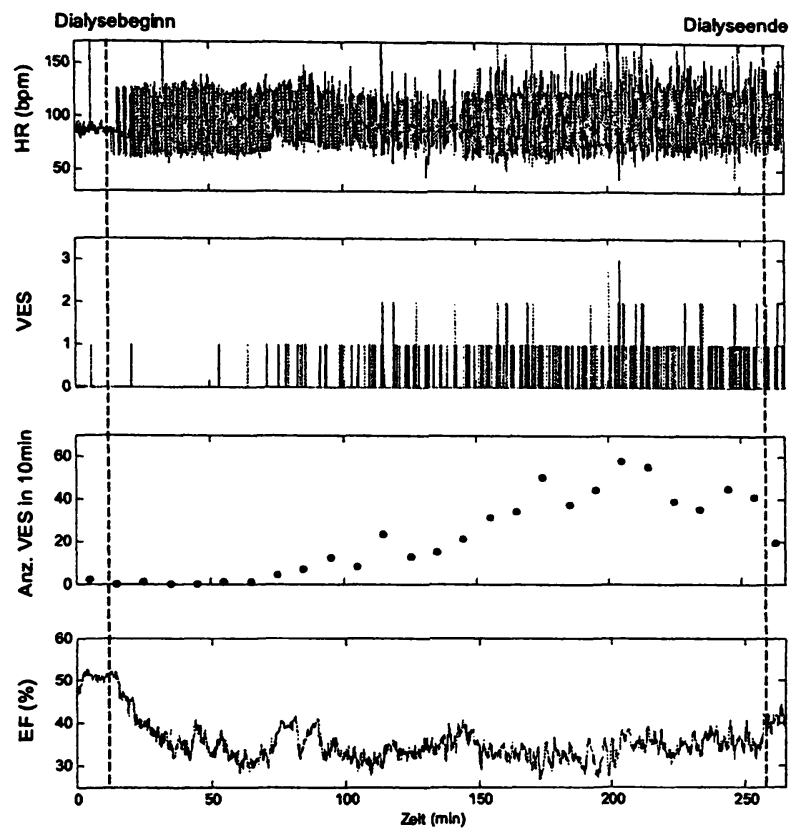

Abbildung 2: Zusammenhang zwischen Ejektionsfraktion und Anzahl ventrikulärer Extrasystolen

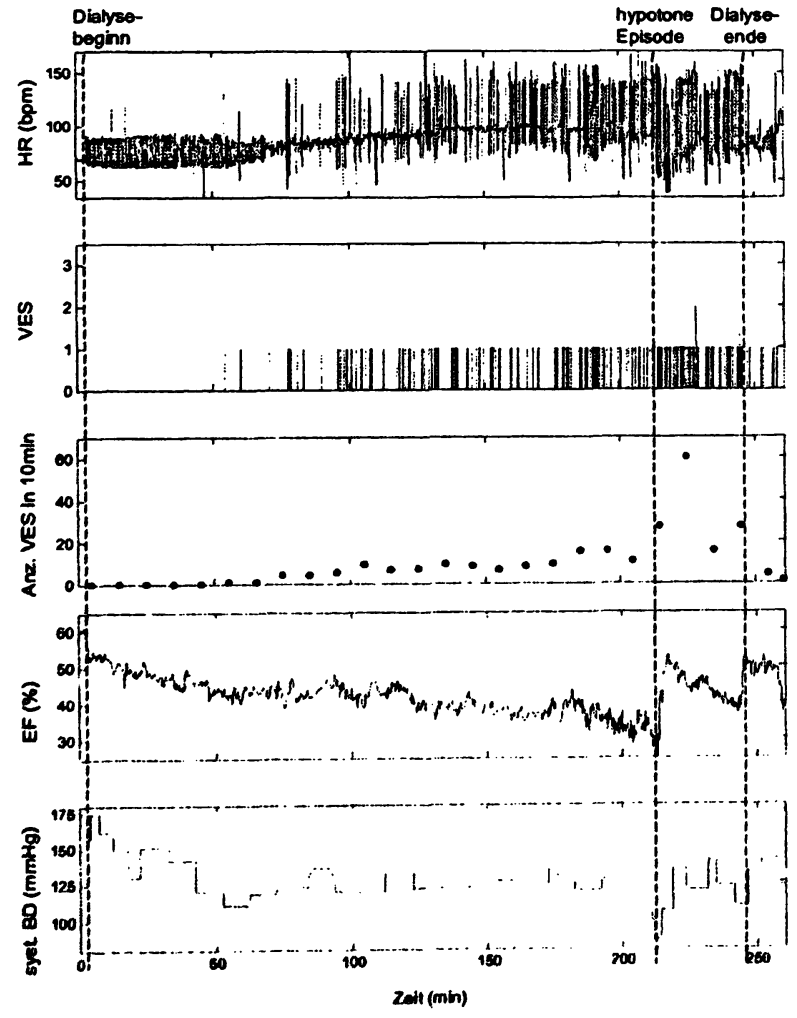

Abbildung 3: Zusammenhang von EF, systolischem Blutdruck und VES-Anzahl bei Dialyseverlauf mit hypotoner Episode
In Abb. 3 sind für die Dialysebehandlung eines 63-jährigen Patienten (Diabetes mellitus IIb, art. Hypertonie WHO II, Z.n. Myokardinfarkt, paVk Std. II) neben der HR und VES-Häufigkeit auch der zeitliche Verlauf von EF und systolischem Blutdruck dargestellt. Nach $210 \mathrm{~min}$ trat eine hypotone Episode auf, der Patient litt unter Übelkeit und Vigilanzstörungen (siehe Marke in Abb. 3). Die durch durch Schocklagerung und Infusion von $250 \mathrm{ml}$ physiologischer Kochsalzlösung erfolgte Intervention führte $\mathrm{zu}$ einer Kreislaufstabilisierung, die sich sowohl in der Normalisierung der EF- und Blutdruckwerte als auch in der Verbesserung des Patientenbefindens verdeutlichte. Eine VES-Häufung trat ca. $12 \mathrm{~min}$ Minuten nach der Blutdruckkrise auf. Nach Beendigung der Dialyse stabilisierten sich die Kreislaufparameter weiter und die VES-Anzahl war ebenfalls rücklaufig.

In Abb. 4 ist anhand des Vergleiches von zwei Dialysebehandlungen eines Patienten (60 Jahre, Diabetes mellitus IIb, art. Hypertonie WHO II, koronare Herzkrankheit) der Einfluss der Elektrolytkonzentration $\left(\mathrm{K}^{+}\right.$und $\mathrm{Ca}^{2+}$ in Serum und Dialysierflüssigkeit) auf eine Arrhythmieentstehung dargestellt.

In Abb. 4 (links) bleibt die VES-Häufigkeit zunächst auf einem niedrigen, klinisch nicht relevanten Wert. Nach $150 \mathrm{~min}$ nimmt sie stark zu und erreicht innerhalb von $60 \mathrm{~min}$ einen Wert von $31 \mathrm{VES}$ pro $10 \mathrm{~min}$. Aufgrund der zu diesem Zeitpunkt gemessenen Serumelektrolytkonzentrationen $\left(\mathrm{K}^{+}: 3,2 \mathrm{mmol} / \mathrm{Ca}^{2+}: 1,31 \mathrm{mmol} / \mathrm{l}\right)$ erfolgte ein Wechsel der verwendeten Dialysierflüssigkeit (siehe Abb. 4). Im Anschluss daran nimmt die VES-Häufigkeit nach einer kurzzeitigen Steigerung auf 36VES/10min kontinuierlich bis zum Dialyseende $a b$.

Im Vergleich dazu ist bei der in Abb. 4 (rechts) dargestellten Dialysebehandlung die VES-Anzahl geringer, ein Wechsel der Dialysierflüssigkeit war nicht erforderlich und die am Behandlungsende erreichten Serumelektrolytkonzentrationen befanden sich an der Grenze zur Hypokaliämie.

\section{Diskussion}

Die hohe Anzahl de novo auftretender ventrikulärer Arrhythmien bestätigt deren dialyseinduziertes Auftreten. Anhand der Messergebnisse konnte gezeigt werden, dass dieses auch für das gehäufte Auftreten von SVES bzw. die Entstehung intermittierender supraventrikulärer Arrhythmien gilt. Im Rahmen unserer Untersuchungen wurden klinisch relevante Arrhythmien beobachtet bei
verminderter Ejektionsfraktion,
- Elektrolytstörungen, vor allem Hyper- und Hypokali- ämie,
- linksventrikulärer Hypertrophic,
- Patienten mit Zustand nach modikamentöscr Candio- version bei vorbestchender absoluter Arrhythmic.

Durch die hohe zeitliche Korrelation der Messwerte konnte ein Zusammenhang awischen stark verminderten lif Werten und einer vermutlich daraus resulticrendell deutlich erhöhten VES-Anzahl nachgewiesen werden. Fiin sturker Abfall der EF wurde in dicsem Zusimmenhang inshesill. dere bei kardial geschädigten Patienten und Anwondung 


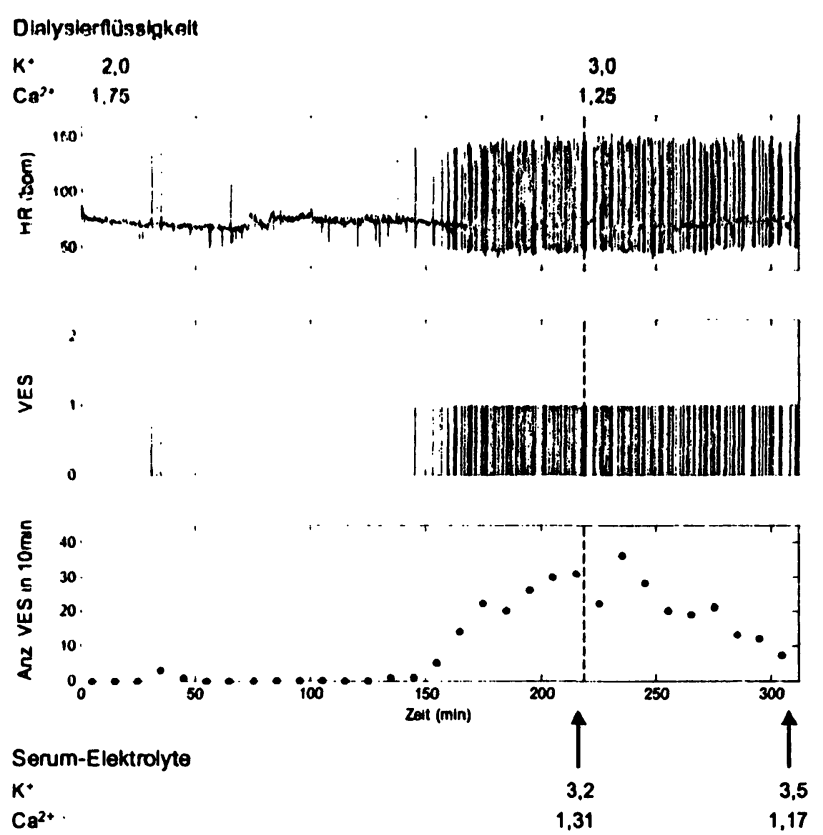

hoher UF-Raten beobachtet. Da die Ejektionsfraktion eine Quantifizierung der linksventrikulären systolischen Funktion ermöglicht, bestätigen die Ergebnisse die aus der Literatur bekannte Arrhythmieneigung bei linksventrikulärer Dysfunktion [1].

Bei den in Abbildung 2 und 3 gezeigten Beispielen löste der EF-Abfall vermutlich über eine Minderdurchblutung des Myokards die Arrhythmieentstehung aus. Dieses zeigt sich u.a. an dem im Vergleich zum EF-Abfall zeitversetzten Auftreten der VES. Zur genauen Klärung dieses $\mathrm{Zu}$ sammenhangs sind weitere Messungen erforderlich.

Weiterhin konnte bei diesen Untersuchungen die in der Literatur diskutierte Arrhythmieentstehung infolge abnormaler Serumelektrolytkonzentrationen [2] bestätigt werden. Wie aus Abb. 4 ersichtlich ist, bewirkt die aufgrund der festgestellten $\mathrm{zu}$ niedrigen Serum- $\mathrm{K}^{+}$-Konzentration erforderliche Anpassung der verwendeten Dialysierflüssigkeit eine zeitversetzte, aber dann kontiuierliche Abnahme der VES-Häufigkeit. Dieser Effekt trat in erster Linie bei kardial geschädigten Patienten auf. Dagegen zeigten Patienten ohne kardiale Begleiterkrankungen eine höhere Toleranz gegenüber geringfügig erniedrigten Serum- $\mathrm{K}^{+}$Konzentrationen (Grenzwert 3,5mmol// [2]), was bei mehreren Patienten anhand der nach Dialyse gemessenen Elektrolytwerte erkennbar war.

Insgesamt zeigen die bisherigen Ergebnisse, dass intradialytische Arrhythmien durch verschiedene Faktoren ausgelöst werden und in der Gruppe der kardial geschädigten Patienten eine hohe Prävalenz aufweisen. Eine intensivere Überwachung dieser Patientengruppe ist deshalb wünschenswert, um bei auftretenden Komplikationen frühzeitig intervenieren zu können. Das hier beschriebene Online-Monitoring gestattet eine derartige Überwachung. Darüber hinaus trägt es zur weiteren Erforschung der Arrhythmieursachen bei, insbesondere da erst durch die komplexe Analyse verschiedener Kreislaufparameter aussage-
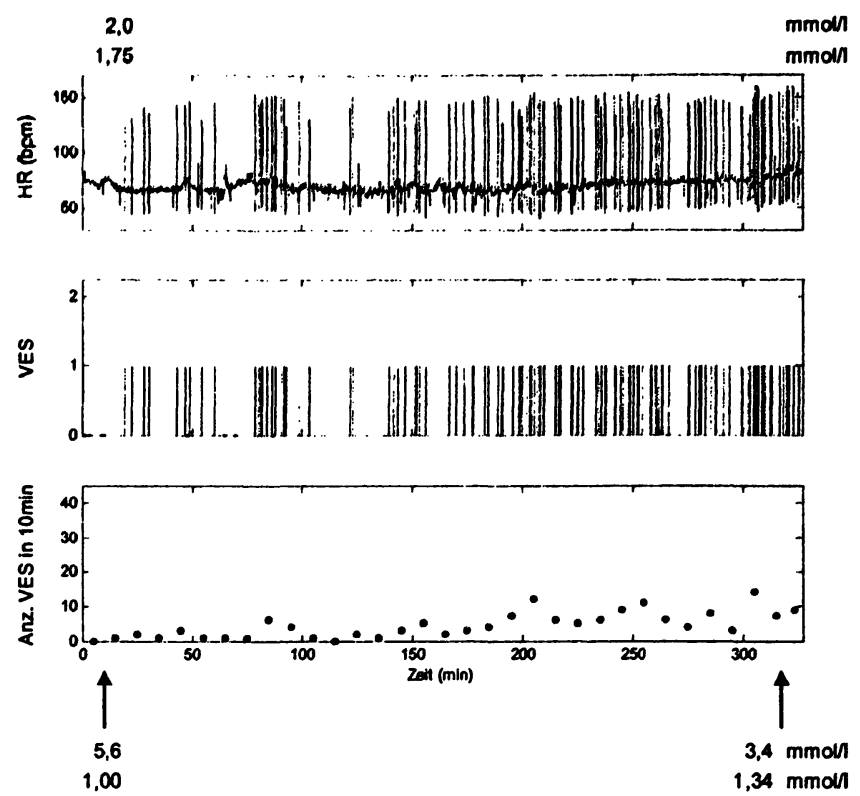

kräftige Informationen über mögliche Entstehungsursachen verfügbar sind.

\section{Schlussfolgerungen}

Das dialyseassoziierte und -aggravierte Auftreten von Extrasystolien und Arrhythmien wird in der klinischen Routine seit Einfuihrung der Bicarbonatdialyse offensichtlich unterschätzt und sollte zukünftig wieder mehr Beachtung finden. Insbesondere bei kardial vorgeschädigten Patienten kann durch das Monitoring eine individuellere Analyse arrhythmieauslösender Faktoren erfolgen. Dieses eröffnet neue Wege zur individuellen Therapieoptimierung, wodurch das Wohlbefinden, die Sicherheit und die Langzeitprognostik der Patienten deutlich verbessert werden können.

\section{Literaturverzeichnis}

[1] "Multicentre, cross-sectional study of ventricular arrhythmias in chronically haemodialysed patients. Gruppo Emodialisi e Patologie Cardiovasculari", Lancet, vol. 2-8606, pp. 305-309, 1988

[2] A. Grassmann, I. Uhlenbusch-Körwer, et al., Composition and Management of Hemodialysis Fluids, Lengerich: Pabst Science Publisher, 2000.

[3] M.A. Saragoça, M.E. Canziani, et al., "Left ventricular hypertrophy as a risk factor for arrhythmias in hemodialysis patients", J. Cardiovasc. Pharmacol., vol. 17Suppl 2, pp. S136-S138, 1991

[4] J.J.G. de Lima, M.L.C. Vieira, et al., "Blood pressure and the risk of complex arrhythmia in renal insufficiency, hemodialysis, and renal transplant patients", Am. J. Hypertens., vol. 12-2 Pt 1, pp. 204-208, 1999

[5] P. Obstoj, B. Schultheiß, et al., "Entwicklung eines online-fahigen Arrhythmiedetektors zur Überwachung von Dialysepatienten", Biomedizinische Technik, vol. 45-Suppl 1, pp. 475-476, 2000 